\title{
A brief review of jorjani's views on Hernias
}

\begin{abstract}
Seyed Esmail Jorjani is one of the greatest Iranian physicians (1042-1136 AC), he has written a book entitled Zakhirah-I Khvarazm Shahi (known as Zachariah). This book is oldest medical encyclopedias written in Persian, it contains a complete subject about hernia. Jorjani described anatomy of anterior abdominal wall and its layers include peritoneum, process vaginalis and tunica vaginalis of testis. He argued about hernias from pathology and etiological point of view and classified abdominal hernias in thousand years ago. European Hernia Society classified the hernias into: lateral, medial and femoral (2007). Birindelli classified anterior abdominal hernias into groin hernias, ventral hernias (2017). Jorjani classified hernia into visceral, flatus, hydrocele (1130AC). The findings of this paper show the hernias subject of Zachariah has no scientific contradiction with new references.
\end{abstract}

Keywords: hernia, iranian traditional, medicine, jorjani, zakhirah-i khvarazm shahi
Volume 4 Issue 5 - 2017

\author{
Reza Mastery Farhani,' Hojjat Allah \\ Abbaszadeh,' Mahtab Teimouri,' Mohsen \\ Bahrami, ${ }^{2}$ Hadi Azimi, ${ }^{3}$ Ramin Pouriran ${ }^{4}$ \\ 'Department of Cell Biology and Anatomical Sciences, Shahid \\ Beheshti University of medical Sciences, Iran \\ ${ }^{2}$ Department of Traditional Medicine, Iran University of Medical \\ Sciences, Iran \\ ${ }^{3}$ Department of English Language Teaching, Shahid Beheshti \\ University of Medical Sciences, Iran \\ ${ }^{4}$ Medical student, Shahid Beheshti University of medical Sciences, \\ Iran
}

Correspondence: Reza Mastery Farahani, Department of Cell Biology and Anatomical Sciences, School of Medicine, Shahid Beheshti University of medical Sciences, Tehran, Iran, Email realmastery@hotmail.com

Received: May 29, 2017 | Published: December 27, 2017

\section{Introduction}

Sir Astley Cooper (19 $9^{\text {th }}$ century surgeon) has a famous science quote which shows the importance of anatomy, "No disease of the human body, belonging to the province of the surgeon, requires in its treatment a greater combination of accurate anatomic knowledge, with surgical skill, than hernia in all its varieties. ${ }^{1}$ The groin hernia is a protrusion in the groin region which is among the oldest recorded afflictions of humanity from beginning to the modern times. ${ }^{2}$ In the Talmud, pointed a kind of scrotal hernia without any mention of possible medical treatment. ${ }^{3}$ The Egyptian Papyrus of Ebers (circa 1552 BC) have an remark about The groin hernia: "when you judge a swelling on the surface of a abdomen.., what comes out . ., caused by coughing. ${ }^{4}$ The mummy of Ramses $5^{\text {th }}$ an Egyptian Pharaoh shows a hernial sac in his groin. ${ }^{5}$ The Greek physicians were trying to find a solution for this worrying disease. ${ }^{6}$ An antique Greeks physicians Celsus provided an operation for inguinal hernia with ligation of the hernial sac and sparing of the testicle with an incision through the scrotum. ${ }^{7}$ Iranian medical scientists, contributed to the development and progress of the anatomical sciences. ${ }^{8}$ Zakhirah written by Seyyed Esmaeil Jorjani is oldest medical encyclopedias written in Persian in the post-Islamic era. Zachariah as Cannon of Avicenna became references of for Persian language physicians. It also was translated into Hebrew, Urdu, and Turkish. Zachariah contains a complete subject about groin hernia. ${ }^{9}$ The study of old Persian medical books such as Zachariah will be beneficial for future historical studies. ${ }^{10}$ The aim of this article is to showing knowledge of anatomy and pathology of Zakhirah and comparing of hernias classification from Jorjani's Views with the same subject in the modern medical books and new groin classification.

\section{Materials and methods}

This is retrospective review on the topic of hernias in Zakhirah, ${ }^{11}$ in this review, the electronic databases were searched to identifying relevant studies. The following search terms were used: abdominal wall hernia, Groin hernias. Jorjani, Zakhirah-I Khvarazm Shahi, Iranian traditional medicine, persian medical history, history of surgery, these key words were searched separately or in other combinations. First, in the results part of the current paper, hernias subject in Zakhirah were translated paragraph by paragraph from Persian into English. The present study uses the closest translations of Zakhirah sentences. Whenever necessary the sentences were summarized, of course some delicate points are added in brackets to clarify the sentences. For better understanding, in the end of discussion part, Phrases from Zakhirah are indicated by $(\mathrm{Z})$ and Phrases of modern references are indicated by $(\mathrm{N})$. Finally the topic of hernias from Zakhirah was compared with modern medical references.

\section{Results}

It is known that coverage of abdomen consists of: skin, muscles and two veils [two covers], the interior veil is tangential to stomach and intestines are called visceral peritoneum. The exterior layer is called the parietal peritoneum since this layer is wider. The advantage of this situation is that the thickness of the two layers do not allow heat to be dismantled in the abdominal viscera and preserve the natural heat of the intestines, there is a collaboration between the viscera and the parietal layer to achieve their tasks, the muscles, outer skin and interlayers fat also is helps to preserve the natural heat. The special benefit of peritoneum is to keep the intestines in their place and to prevent them from unfolding and descending in empty spaces. Extension and tension of peritoneum is one of its benefits. 
This peritoneum descends to groin and inguinal region on right and left side and two vents [deep inguinal foramens] are made in it [in during the gestation] small canal process vaginalis which descends from the vents in each side almost to the testis and opens there, then each is closed again forming a sac around each testis [that is Tunica vaginalis].

If someone jumps hard or suffers from a sever situation, shouts hard moves a heavy objects or any force, or strains like these are exerted on him, the peritoneum by the effective forces becomes torn and perforated. If this perforation was parallel to umbilicus or upper or lower than it and the greater omentum entering the umbilicus by the perforation, this kind of hernia is umbilical and Para umbilical hernias.

If the deep inguinal foramen which is made in the bottom of peritoneum tears, the intestine and the greater omentum descends from it, this foramen may be small or big, if the foramen is small; the intestine does not descend lower than femoral region. This type of hernias is called incomplete inguinal hernia .If the foramen [deep inguinal foramen] is big, intestine descends to the scrotum sac and is called herniation of intestine to scrotum, nowadays named scrotal hernia. In some cases, the cause of descending of intestine to inguinal region and scrotum is not tearing in peritoneum but moisture aggregation near the foramens. One or both of these foramens [right and left deep inguinal foramen] weaken after soaking by this moisture and extend by small forces, the moisturized intestine then descends from these foramens and incomplete inguinal hernia and scrotal hernia is made.

These hernias are of three type One type is, as mentioned earlier scrotal hernia, incomplete inguinal hernia, umbilical and Paraumbilical hernias. The second type is when wind comes to scrotum and makes it like pumpkin which is called flatus hernia. The third type is made when the fluid descends to scrotum and is called hydrous hernia or hydrocele. The women may be acquired the umbilical and Paraumbilical hernias and incomplete inguinal hernia.

The sign of umbilical and Paraumbilical hernias is the withdrawal of the prominent mass in supine position and becoming prominent in sitting position. Also the mass is reduced by hand pressure and comes out by deleting the pressure. The sign of incomplete inguinal hernia is the same. Gargouillement in scrotum is the sign of complete inguinal hernia especially in case of eating the meals that produce flatus or difficulty in digestion or walking long. This kind is reducible by hand pressure but sometimes it descends out and cannot be reduced then scrotum becomes hard as a stone and cause a lot of pain suffering till becomes reduced $(\mathrm{Z})$.

\section{Discussion}

Even today, hernia genesis is still an exciting issue for practitioners and scientists. Many scientists are constantly engaged with studies on this topic, looking to establish all of the factors involved in the development of the disease. ${ }^{12}$ The abdominal wall consists of skin, superficial fascia, fat, muscles, transversalis fascia, and the parietal peritoneum. The peritoneum has potential spaces produced by the visceral and parietal peritoneum which are important to realize. ${ }^{13}$ It should be known that coverage of abdomen consists of: skin, muscles and two veils (two covers), the interior veil is tangential to stomach and intestines are called visceral peritoneum. The exterior layer is called the parietal peritoneum $(\mathrm{Z})$. The repair of inguinal hernia requires an understanding of the embryology of descent of the testes and the formation of the processes vaginalis. The peritoneal layer which enveloping testis and named tunica vaginalis. ${ }^{14}$ This peritoneum descends to groin and inguinal regions and two vents [deep inguinal foramens] are made in it, as two canalicullus named process vaginalis which descends from the vents in each side almost to the testis and opens there, then each is closed again forming a sac around each testis as Tunica vaginalis $(Z)$. This paragraph is an exact explanation of inguinal canal and vaginal process and the tunica vaginalis. In spite of the existence this document, what is mentioned in the new resources is: in 1790, John Hunter gave the first account of testicular descent and structure of the gubernaculums of testis. ${ }^{15}$

The mechanism of Acquired inguinal hernia and the cause of most acquired inguinal hernias is undefined and not well known but body exercises, coughing, straining, external pressure, geriatric, surgical operations may be the main causes. From an anatomical and embryological aspect, damage weakening of the inguinal triangle, patent processes vaginalis, failure of the shutter mechanism, and dilatation of the inguinal ring, are important factors. ${ }^{16}$ The acquired Hernia may be caused by conditions that increase the pressure in the abdominal cavity (obesity, coughing, straining), from previous surgical procedure (incisional hernia) or from typical trauma, preperitoneal fat, or the greater omentum protrudes through the hernial defect. If the hernia is left untreated, bowel loops may be incorporated into the hernia. ${ }^{17}$

If someone jumps hard or suffers from a sever situation, shouts hard, moves a heavy objects or any force, or strains like these are exerted on him, then hernia occurs (Z). In indirect inguinal hernia, intestine passes through the deep ring of inguinal canal and passes the canal. If the hernias extend through superficial inguinal ring, it is called complete inguinal hernia. In men, intestine enters the scrotum and so is called scrotal hernia, and if it does not extend through superficial inguinal ring, it is called incomplete inguinal hernia. ${ }^{18}$ Zakhirah states the same procedure: There, it is mentioned that if the intestine does not descend lower than inguinal region, it is called incomplete inguinal hernia and if the intestine reaches the scrotum, it is scrotal or complete inguinal hernia (Z). A hernia is reducible if it occurs intermittently (such as standing position) and can be pushed back into the abdominal cavity, and irreducible if it remains permanently outside the abdominal cavity. A reducible hernia is usually a longstanding condition. ${ }^{19}$ Jorjani in Zakhirah writes: The sign of umbilical and Paraumbilical hernias is the withdrawal of the prominent mass in supine position and becoming prominent in sitting position. Also, the mass is reduced by hand pressure and comes out by removing the pressure. The sign of incomplete inguinal hernia is the same. Gargouillement in scrotum is the sign of complete inguinal hernia, especially in case of eating the meals that produce flatus or difficulty in digestion or lengthy walk. This kind of hernia is reducible by hand pressure but sometimes it descends out and cannot be reduced then scrotum becomes hard as a stone and causes a lot of pain suffering till it becomes reduced $(Z)$.

Sex, age, region, sites or location, size, function, surgery, recurrence, etiological and epidemiology are factors for abdominal wall hernias classification. ${ }^{20}$ On the basis of above mentioned factors in recent time there is around 10 kind of hernias classification. ${ }^{21}$ Gilbert use an anatomic and functional classification..$^{22}$ Nyhus use anatomical aspect $\mathrm{t}^{23}$ and Schumpelick classification factors are size of the defect, position of hernia, the site of the incision and the number 
of previous repairs. ${ }^{24}$ The European Hernia Society (EHS) classified the hernias to lateral medial and femoral types and it is an easy to remember kind. ${ }^{25}$ The new classification of Abdominal hernias is: groin hernias (femoral or inguinal) and ventral hernias (umbilical, epigastric, Spigelian, lumbar, and incisional) ${ }^{26}$ Jorjani in Zakhirah classified hernia into three types. Type 1 includes scrotal hernia, incomplete inguinal hernia, and umbilical and Paraumbilical hernias. Type 2 includes flatus hernia, and type 3 includes hydrocele.

\section{Conclusion}

Jorjani described anatomy of anterior abdominal wall and its layers includes peritoneum, process vaginalis and tunica vaginalis of testis. He described anatomy, pathology, etiology anterior abdominal hernias The EHS classification is directional and the Birindelli et al., ${ }^{26}$ classification is regional and surgical. Therefore, Jorjani's priority is evident in the hernia classification. There is no scientific contradiction between these categories, only that EHS classification has regional and directional basis, Arianna Birindelli is anatomical and surgical, and Jorjani classification is anatomical and material basis. Regarding what is stated, it is obvious that scientific information in Zakhirah is approved by the new information even after about 1000 years. So, it is an initiative factor for doing more studies on other topics of this precious textbook.

\section{Acknowledgements}

None.

\section{Authors' contribution}

All authors contributed to the design and writing of the article.

\section{Financial disclosure}

None.

\section{Funding/Support}

None.

\section{Conflict of interest}

Author declares that there is no conflict of interest.

\section{References}

1. Maddern G, Karatassas A, Hensman C. Pain or gain: new innovations and trends in hernia repair. ANZ J Surg. 2009;79(11):778-780.

2. Onuigbo WIB, Njeze GE. Inguinal Hernia. A Review. J Surg Oper Care. 2016;1(2):202.

3. Pournaropoulos GK, Emmanuel K Hippocrates. All His Works. Athens, Greece; 1967. 163p.

4. Lau WY. History of Treatment of Groin Hernia. World $J$ Surg. 2002;26(6):748-759.

5. Gupta RK, Bhuvan, Shahi KS, et al. Lichenstein tension free mesh hernioplasty: a prospective study. $J$ of Evolution of Med and Dent $S c i$. 2014;3(67):14424.

6. Andrzej L Komorowski. History of the inguinal hernia repair. UK: In Tech; 2014.
7. Johnson J, Roth JS, Hazey JW, et al. The history of open inguinal hernia repair. Curr Surg. 2004;61(1):49-52.

8. Shoja MM, Tubbs RS. The history of anatomy in Persia. $J$ Anat. 2007;210(4):359-378

9. Moattar F, Shams Ardekani MR, Ghannadi A. The life of jorjani: one of the persian pioneers of medical encyclopedia compiling: on the occasion of his 1000th birthday anniversary (434, AH-1434, AH). Iran Red Crescent Med J. 2013;15(9):763-766.

10. Bahrami M, Farahani RM, Nazem E, et al. The body organs and their reconstruction power (regeneration) from the viewpoint of iranian-islamic physicians. Iran Red Crescent Med J. 2014;16(3):e15193.

11. Sirjani S. Introduction. In: Zakhireye Khwaram Shahi (Treasure of Khwarazm Shah) [in Persian]. Entesharat-e Bonyad-e Farhand-e Iran, Tehran, Iran; 1976. p. 532-533.

12. Amato G, Marasa L, Sciacchitano T, et al. Histological findings of the internal inguinal ring in patients having indirect inguinal hernia. Hernia. 2009;13(3):259-262.

13. Le Ott. Patterns of peritoneal spread of tumor in the abdomen and pelvis World J Radiol. 2013;5(3):106-112.

14. Wang KS, Papile LA, Baley JE, et al. Assessment and management of inguinal hernia in infants. Pediatrics. 2012;130(4):768-773.

15. Coveney D, Shaw G, Hutson JM, et al. The development of the gubernaculum and inguinal closure in the marsupial Macropus eugenii. $J$ Anat. 2009;201(3):239-256.

16. Kawaguchi M, Kadono Y, Shimada M, et al. Laparoscopic observations in acquired inguinal hernia before and after robotic-assisted laparoscopic prostatectomy. Surgical Science. 2014;5:170-175.

17. Lassandro F, Iasiello F, Pizza NL, et al. Abdominal hernias: Radiological features. World J Gastrointest Endosc. 2011;3(6):110-117.

18. Garriga V, Serrano A, Marin A, et al. US of the tunica vaginalis testis: anatomic relationships and pathologic conditions. Radiographics. 2009;29(7):2017-2032.

19. Jenkins JT, O’Dwyer PJ. Inguinal hernias. BMJ. 2008;336(7638):269-272.

20. David C Brooks, Michael Rosen, Wenliang Che. Overview of abdominal wall hernias in adults, Up-to-date. 2017

21. Kulacoglu H. Current options in inguinal hernia repair in adult patients. Hippokratia. 2011;15(3):223-231.

22. Gilbert AI. An anatomic and functional classification for the diagnosis and treatment of inguinal hernia. Am J Surg. 1989;157(3):331-333.

23. Nyhus LM, Condon RE, Harkins HN. Clinical experience with preperitoneal hernia repair for all types of hernia of the groin. Am J Surg. 1960;100:234-244.

24. Schumpelick V, Treutner KH, Arlt G. Classification of inguinal hernias. Chirurg. 1994;65(10):877-879.

25. Miserez M, Alexandre J, Campanelli G, et al. The European hernia society groin hernia classification: simple and easy to remember. Hernia 2007;11(2):113-116

26. Birindelli A, Sartelli M, Di Saverio S, et al. 2017 update of the WSES guidelines for emergency repair of complicated abdominal wall hernias. World J Emerg Surg. 2017;12:37. 